\title{
TROPICAL CYCLONE VECTOR WINDS FROM C-BAND DUAL-POLARIZATION SYNTHETIC APERTURE RADAR
}

\author{
Biao Zhang ${ }^{1}$, William Perrie ${ }^{2}, Y_{i j u n} \mathrm{He}^{1}$, Zhongfeng Qiu ${ }^{1}$, Jie Guo ${ }^{3}$ \\ Nanjing University of Information Science and Technology, Nanjing, 210044, China \\ Bedford Institute of Oceanography, Dartmouth, B2Y 4A2, Canada \\ Yantai Institute of Coastal Zone Research, Chinese Academy of Sciences, Yantai, 264003, China
}

\begin{abstract}
We presents a new method for retrieving tropical cyclone vector winds using C-band dual-polarization SAR observations. The co-polarized geophysical model function (CMOD5.N) and a cross-polarized wind speed retrieval model for dual-polarization (C-2POD) are employed to construct a cost function. minimization of the cost function allows optimum estimates for the wind speeds and directions. The wind direction ambiguities are removed by a parametric two dimensional sea-surface inflow angle model.
\end{abstract}

Index Terms - Tropical cyclone, Synthetic aperture radar, Vector winds, Dual-polarization

\section{INTRODUCTION}

Over the last few years, great efforts have been devoted to investigate hurricane winds with conventional singlepolarization SAR data. A typical C-band RADARSAT-1 SAR image of Hurricane Ivan on September 10, 2004 was used to investigate the feasibility of retrieval of hurricane force winds utilizing conventional geophysical model functions, such as CMOD4 and CMOD5 [1]. To avoid the influence of inaccurate wind directions on wind speed inversion, a new algorithm was proposed to retrieve hurricane wind speed and wind direction simultaneously, based on the primary hurricane structure characteristics [2]. However, this method needs to be modified in some circumstances, for example, for degraded hurricanes which tend to gradually lose their circular structure, and for SAR images not containing the hurricane eye.

Recently, C-band cross-polarized ocean backscatter has been documented as being insensitive to the wind direction or the radar incidence angle, and quite linear with respect to the wind speed, and thus can be used to directly retrieve wind speeds [3]. An empirical C-band Cross-Polarization Ocean (C-2PO) backscatter model was proposed to retrieve high winds from satellite SAR data, which eliminates the need for external wind direction and radar incidence angle inputs and provides a linear response at high wind speeds for hurricanes [4]. Compared to single-polarization (HH or
VV) SAR, dual-polarization (VV+VH) SAR can simultaneously measure ocean backscatters with different scattering characteristics, which provides an opportunity to investigate hurricane wind vector retrievals. The objective of this study is to develop a method to retrieve tropical cyclone vector winds with C-band dual-polarization SAR observations.

\section{DATASET}

We collected 648 RADARSAT-2 dual-polarization (VV, VH) SAR images for the time interval November 2008 to March 2011, with 39 in situ National Data Buoy Center (NDBC) buoy observations in the Gulf of Alaska, and off the East and West Coasts of the United States. The spatial and temporal windows for the collocations are required to be less than $10 \mathrm{~km}$ and $30 \mathrm{~min}$, respectively. In addition to collocations between SAR images and buoy observations mentioned above, we also collected SFMR and $\mathrm{H}^{*}$ Wind data, which were collocated with SAR images of Hurricanes Earl and Ike acquired at 22:59 UTC September 2, 2010 and 23:56 UTC September 10, 2008, respectively. SFMR measurements over Hurricane Earl were obtained during 22:30 23:30 UTC September 2, 2010. SFMR can potentially provide along-track mapping of surface wind speeds at relatively high spatial $(1.5 \mathrm{~km})$ resolution. For Hurricane Ike, $\mathrm{H}^{*}$ Wind data were acquired at 01:30 UTC September 11, 2008. The spatial resolution of $\mathrm{H}^{*} \mathrm{Wind}$ data is $6 \mathrm{~km}$. In total, the dataset consists of 1845 collocated pairs (buoy: 884 , SFMR: $348, \mathrm{H}^{*}$ Wind: 613 ) of NRCS in $\mathrm{VH}$ polarization and wind speeds, which were used to develop the wind speed retrieval model for dual-polarization SAR.

\section{METHOD}

In the present study, we incorporate SFMR-measured surface winds and also $\mathrm{H}^{*} \mathrm{Wind}$ data. In total, there are 633 wind speeds above $20 \mathrm{~ms}^{-1}$, and the highest wind speed is $39.7 \mathrm{~ms}^{-1}$ as shown in Fig. 1, providing an opportunity to develop a high wind speed retrieval model for dualpolarization SAR observations. Fig. 1 shows the relation 
between RADARSAT-2 measured VH-polarized NRCS $\left(\sigma_{V H}^{0}\right)$ and collocated observed $10-\mathrm{m}$ neural wind speed $\left(U_{10}\right)$, from buoys, SFMR measurements, and $\mathrm{H}^{*}$ Winds data. We find that $\sigma_{V H}^{0}$ is not sensitive to the radar incidence angle or wind direction, but is dependent on wind speed. We use nonlinear least squares to derive a model relating $\sigma_{V H}^{0}$ to $U_{10}$, as follows:

$$
\sigma_{V H}^{0}=0.332 * U_{10}-30.143
$$

In Eq. 1 , the units of $\sigma_{V H}^{0}$ and $U_{10}$ are $\mathrm{dB}$ and $\mathrm{ms}^{-1}$, respectively. The correlation coefficient between observed $\sigma_{V H}^{0}$ and simulated $\sigma_{V H}^{0}$ with Eq. 1 is 0.93 and the relation is independent of external wind direction or radar incidence angles. Therefore, we have developed a $\underline{\mathrm{C}}$-band $\underline{\text { Cross- }}$ Polarized Ocean surface wind retrieval model for Dualpolarization SAR, hereafter denoted as C-2POD. Moreover, as dual-polarization SAR provides simultaneous co- and cross-polarized ocean backscatter with different scattering characteristics, we can potentially retrieve wind vectors.

In order to simultaneously retrieve wind speed and direction, we construct a cost function involving both the co-polarized geophysical model function (CMOD5.N) and $\mathrm{C}-2 \mathrm{POD}$. The cost function is:

$$
J(i, j)=\left[\sigma_{V V}^{m}(i, j)-\sigma_{V V}^{o}(i, j)\right]^{2}+\left[\sigma_{V H}^{m}(i, j)-\sigma_{V H}^{o}(i, j)\right]^{2}
$$

where $\sigma_{V V}^{m}$ and $\sigma_{V H}^{m}$ are simulated and $\sigma_{V V}^{o}$ and $\sigma_{V H}^{o}$ are observed NRCS in VV and VH polarizations, respectively. Here, $i$ and $j$ are the line and column pixel locations in the SAR image. We minimize the cost function at each pixel, using a nonlinear least squares technique, and obtain optimum estimates for wind speed, and direction, with ambiguities.

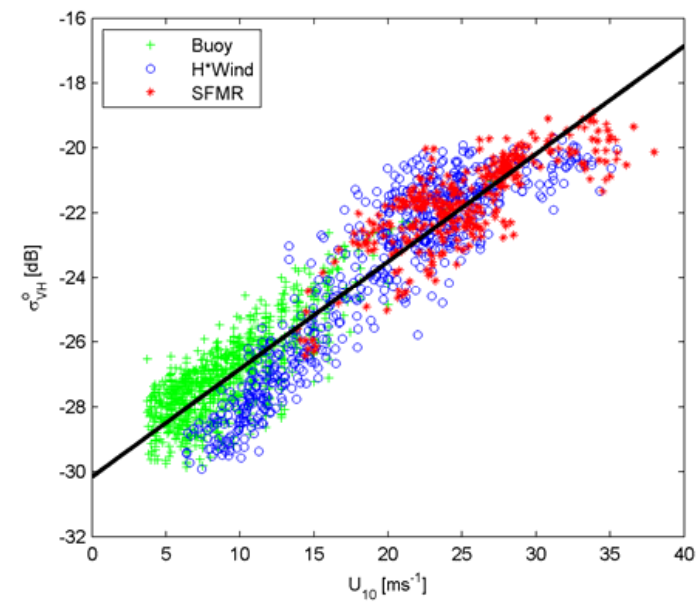

FIG. 1. Mean $\sigma_{v H}^{0}$ from RADARSAT-2 dual-polarization

SAR versus in situ measured $U_{10}$ from buoys, SFMR data and $\mathrm{H}^{*}$ Wind; the correlation coefficient between SAR observed $\sigma_{V H}^{0}$ and simulated $\sigma_{V H}^{0}$ with Eq. 1 is 0.93 .

Due to the friction between hurricanes and the ocean surface, the winds inside the hurricane rotate counterclockwise and the inflow angle is towards the storm's center, in the northern hemisphere. Recent investigation [5] has showed that the mean inflow angle in hurricanes is in the range $-22.6 \pm 2.2^{\circ}$ with $95 \%$ confidence, from analysis of near-surface $(10-\mathrm{m})$ inflow angles using wind vector data from over 1600 quality-controlled Global Positioning System dropwindsondes deployed by aircraft on 187 flights in 18 hurricanes. They proposed an analytical parametric model for the surface inflow angle $\alpha_{S R}$, which requires, as inputs, the storm motion speed, maximum wind speed, and radius of maximum wind, according to the relationship:

$$
\begin{aligned}
& \alpha_{S R}\left(r^{*}, \theta, V_{\max }, V_{s}\right)=A_{\alpha 0}\left(r^{*}, V_{\max }\right)+ \\
& A_{\alpha 1}\left(r^{*}, V_{s}\right) \cos \left[\theta-P_{\alpha 1}\left(r^{*}, V_{s}\right)\right]+\varepsilon
\end{aligned}
$$

where $V_{\max }$ and $V_{s}$ are maximum wind speed and storm motion speed, respectively. Here, $r^{*}$ is the normalized radial distance with radius of maximum wind speed $\left(r^{*}=r / R_{\max }\right), \varepsilon$ is model error, and $A_{\alpha 0}, A_{\alpha 1}$ and $P_{\alpha 1}$ are functions that are described in [5].

In this study, the model-estimated inflow angle can be used to select the 'optimum wind direction' from the multiple of possible wind direction solutions. At each pixel location, the wind alias nearest to the counter-clockwise tangential direction, less than estimated inflow angle (toward the storm center), is chosen as the correct wind direction. In order to obtain the tangential direction at each pixel location, we use the approach [6] to determine the location of the center of the hurricane eye. Since C-2POD is not saturated under high winds, we can derive maximum wind speed from the retrieved wind speeds. Thus, the radius of maximum wind can be obtained by estimating of the distance between the hurricane eye center and the maximum wind speed location. Storm motion speed can be acquired from National Hurricane Center (NHC) 6-hourly Best-Track data. Therefore, the inflow angle is easily calculated using hurricane intensity and the motion parameters using the parametric inflow angle model. SAR wind direction determination, among the possible aliases, is therefore easily accomplished making use of the inflow angle at each pixel location.

\section{RESULTS AND DISCUSSIONS}

Figs $2 \mathrm{a}$ and $2 \mathrm{~b}$ show a RADARSAT-2 SAR image of Hurricane Bill in VV and VH polarizations, respectively. The cost function uses co- and cross-polarized wind speed retrieval models (CMOD5.N and C-2POD), as well as 
NRCS in VV and $\mathrm{VH}$ polarizations and radar incidence angles, to obtain the wind speed and direction, with ambiguities. We can easily derive the hurricane intensity (maximum wind speed) and radius of maximum wind from the retrieved wind speeds. They are $34.8 \mathrm{~ms}^{-1}$ and $40.1 \mathrm{~km}$, respectively. The motion speed of Hurricane Bill is given by the NHC 6-hourly Best-Track data, which is about $9.4 \mathrm{~ms}^{-1}$. The optimum wind speeds associated with the minimum cost function are shown in Figure 2c. The storm-relative inflow angles are calculated using the hurricane intensity, radius of maximum wind and storm motion speed, with the parametric model [5]. As shown in Figure 3, the estimated inflow angles have mean value of $-22.5^{\circ}$. As mentioned previously, the wind alias closest to the counter-clockwise tangential direction less than the estimated inflow angle (toward the storm center) is selected as the optimum wind direction. Thus, we remove the ambiguity in the solutions and obtain the final unique wind directions, which are shown in Figure 2d.
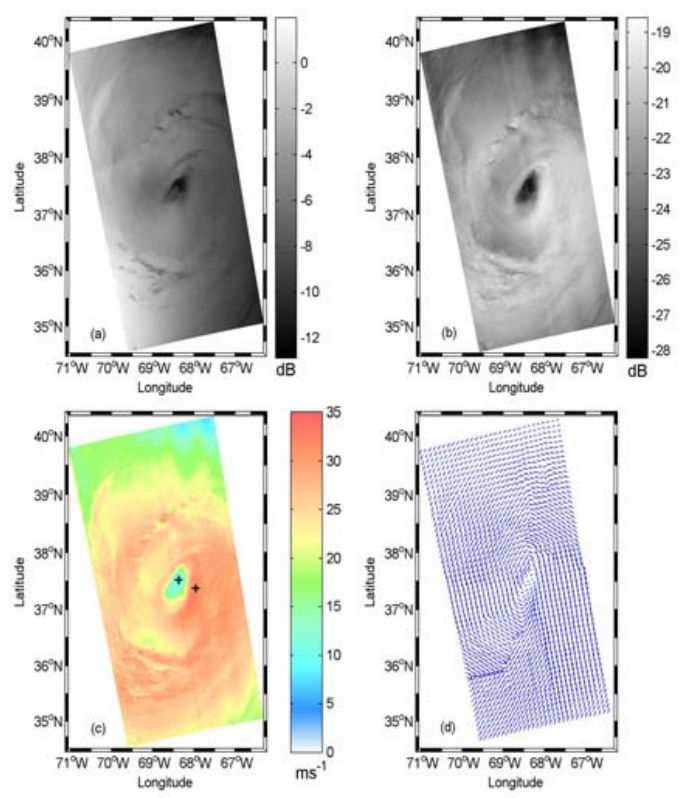

FIG. 2. RADARSAT-2 dual-polarization SAR image acquired over Hurricane Bill at 22:26 UTC on August 22, 2009 showing: (a) VV polarization and (b) VH polarization, where the colorbar shows sigma-naught in VV polarization $\left(\sigma_{\mathrm{VV}}^{\mathrm{o}}\right)$ and in $\mathrm{VH}$ polarization $\left(\sigma_{\mathrm{VH}}^{\mathrm{o}}\right)$ in $\mathrm{dB}$, respectively. (c) SAR-retrieved wind speeds. Colorbar shows wind speeds at 10-m height $\left(\mathrm{U}_{10}\right)$ in $\mathrm{ms}^{-1}$, and (d) SAR-retrieved wind directions without ambiguities. RADARSAT-2 Data and Product (C) MacDonald, Dettwiler and Associates Ltd, - All Rights Reserved. The locations of HEC and $V_{\max }$ are indicated as the black plus $(+)$ in Fig. 2c.

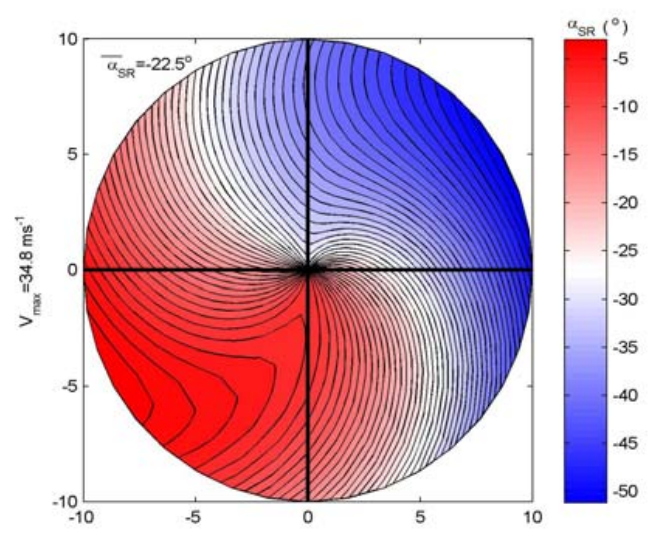

FIG. 3. Storm-relative inflow angle $\left(\alpha_{S R},{ }^{\circ}\right)$ computed by the parametric model for Hurricane Bill motion speed of $V_{s}=9.4 \mathrm{~ms}^{-1}$, and intensities of $V_{\max }=34.8 \mathrm{~ms}^{-1}$. Storm direction is toward the top of the figure.

Since no available SFMR and $\mathrm{H}^{*}$ Wind data can be collocated with SAR images of Hurricanes Bill and Bertha at 22:26 UTC on August 22, 2009 and 10:14 UTC on July 12, 2008, we match QuikSCAT winds with SAR retrievals according to the nearest distance criteria, to evaluate the accuracy of the proposed hurricane wind vector method. QuikSCAT-measured winds for Hurricanes Bill and Bertha are acquired at 22:54 UTC on August 22, 2009 and 09:42 UTC on July 12, 2008, respectively. For Hurricanes Bill and Bertha, the time difference between SAR observations and QuikSCAT measurements are only 28 and 32 minutes, respectively.

For Hurricane Bill, we compare the wind speeds from C2POD and QuikSCAT in Fig. 4a. They show good consistency even for high wind speeds $\left(>20 \mathrm{~ms}^{-1}\right)$. Fig. $4 \mathrm{~b}$ shows the wind speeds from C-2POD versus those from CMOD5.N. It is shown that CMOD5.N wind speeds are underestimated, compared to C-2POD values, which is possibly caused by saturation of the NRCS in VV polarization, under high wind conditions and inaccuracy in the external wind direction input. Fig. 4c shows the SARretrieved wind directions versus QuikSCAT measurements. Rain has a significant impact on QuikSCAT wind speeds, but not on wind directions, except for very high rain rates [7]. We quantitatively estimate the bias, and centered root mean square error (RMSE), based on the formula proposed by [8]. Compared to conventional RMSE, the centered RMSE is not dependent on the bias. We calculate the wind direction correlation following the vector correlation $\left(\rho_{v}^{2}\right)$ approach [9], where $\rho_{v}^{2}=2$ and $\rho_{v}^{2}=0$ are related to perfect correlation and zero correlation, respectively. 


\section{CONCLUSIONS}

We have presented a method to simultaneously determine hurricane wind speed and direction with C-band RADARSAT-2 dual-polarization SAR observations. The co-polarized geophysical model function (CMOD5.N) and a cross-polarized wind speed retrieval model for dualpolarization (C-2POD) are employed to construct a cost function. Minimization of the cost function allows optimum estimates for the wind speeds and directions. The wind direction ambiguities are removed using a parametric two dimensional sea-surface inflow angle model.
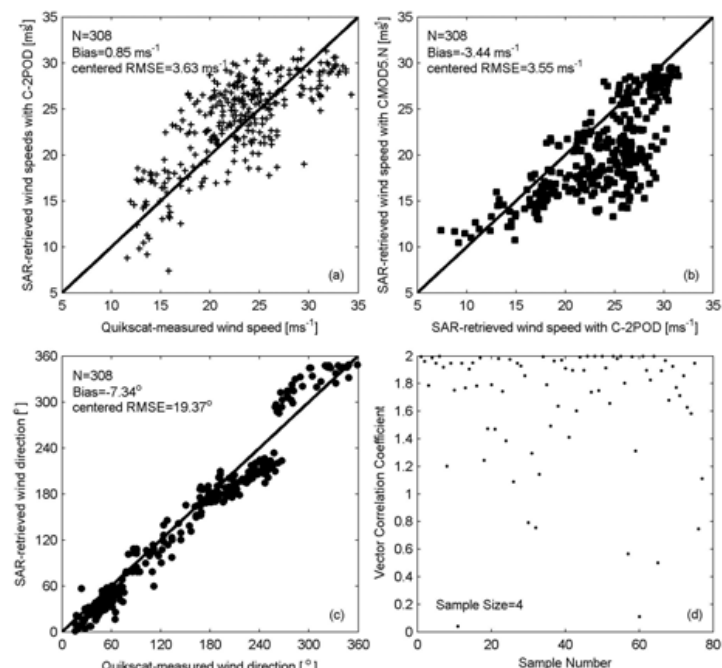

FIG. 4. (a) SAR-retrieved wind speeds from the C-2POD model versus QuikSCAT-measured wind speeds, (b) SARretrieved wind speeds from the CMOD5.N model versus from the C-2POD model, (c) SAR-retrieved wind directions versus QuikSCAT-measured wind directions, (d) Vector correlation of wind directions from SAR and QuikSCAT (sample size is 4). Hurricane Bill winds from SAR and QuikSCAT are acquired at 22:26 UTC on August 22, 2009 and 22:54 UTC on August 22, 2009, respectively.

\section{ACKNOWLEDGEMENTS}

The authors thank the Canadian Space Agency (CSA) for providing RADARSAT-2 dual-polarization SAR hurricane imagery, and NOAA HRD and NDBC for supplying SFMR and buoy observations, respectively. This work was supported by the Chinese National High Technology Research and Development (863) Program grant 2013AA09A505, and Natural Science Youth Foundation of Jiangsu Province grant BK2012467, and National Natural Science Foundation of China grant 41176160.

\section{REFERENCES}

[1] J. Horstmann, D.R. Thompson,F. Monaldo, S. Iris, and H. C. Graber, "Can synthetic aperture radars be used to estimate hurricane force winds?," Geophys. Res. Lett., 32, L22801, 2005, doi:10.1029/2005GL023992.

[2] H. Shen, Y. He, and W. Perrie, "A new hurricane wind retrieval algorithm for SAR images," Geophys. Res. Lett., 33, L21812, 2006, doi:10.1029/2006GL027087.

[3] B. Zhang, W. Perrie, and Y.He, "Wind speed retrieval from RADARSAT-2 quad-polarization images using a new polarization ratio model," J. Geophy. Res, 116, C08008, 2011, doi:10.1029/2010JC006522.

[4] B. Zhang, W. Perrie, "Cross-polarized synthetic aperture radar: a new potential technique for hurricanes," Bull. Amer. Meteor. Soc, 93, 531-541, 2012, doi:10.1175/BAMS-D-11-00001.1.

[5] J. Zhang, and E. W. Uhlhorn, "Hurricane sea-surface inflow angle and observation-based parametric model," Mon. Wea. Re , 140, 3587-3605, 2012, doi:10.1175MWR-D-11-00339.1.

[6] Y. Du and P. W. Vachon, "Characterization of hurricane eyes in RADARSAT-1 images with wavelet analysis," Can. J.Remote. Sen, vol. 29, pp. 491-498, 2003.

[7] L. Ricciardulli and F. Wentz, "Reprocessed QuikSCAT (V04) wind vectors with $\mathrm{Ku}-2011$ geophysical model function, "Remote Sens. Syst. Tech. Rep. 043011, Remote Sens. Syst., Santa Rosa, Calif.

[8] T.-Y. Koh, S. Wang, and B. C. Bhatt, "A diagnostic suite to assess NWP performance. J. Geophys. Res., 117, doi:10.11029/2011JD017103, 2012.

[9] D. Crosby, L. C. Breaker, and W. H. Gemmill, "A proposed definition for vector correlation in geophysics: theory and application. J. Atmos. Oceanic Technol., 30, 395-428, 1993. 